\title{
Prognosis after liver transplantation in patients treated with anti- PD-1 immunotherapy for advanced hepatocellular carcinoma: case series
}

\author{
Zhitao Chen ${ }^{1,2,3 \#}$, Xitao Hong ${ }^{1,2,3 \#}$, Tielong Wang ${ }^{1,2,3}$, Yiwen Guo ${ }^{1,2,3}$, Changjun Huang ${ }^{1,2,3}, \mathrm{Muqi}^{\mathrm{Li}^{4}}$, \\ Xiaoshun $\mathrm{He}^{1,2,3}$, Weiqiang Ju ${ }^{1,2,3}$, Maogen Chen ${ }^{1,2,3}$
}

${ }^{1}$ Organ Transplant Center, First Affiliated Hospital of Sun Yat-sen University, Guangzhou, China; ${ }^{2}$ Guangdong Provincial Key Laboratory of Organ Donation and Transplant Immunology, Guangzhou, China; ${ }^{3}$ Guangdong Provincial International Cooperation Base of Science and Technology (Organ Transplantation), Guangzhou, China; ${ }^{4}$ Department of Liver Surgery, The First Affiliated Hospital of Sun Yat-sen University, Guangzhou, China

Contributions: (I) Conception and design: M Chen, W Ju; (II) Administrative support: X He; (III) Provision of study materials or patients: Z Chen, X Hong; (IV) Collection and assembly of data: Z Chen, X Hong, T Wang; (V) Data analysis and interpretation: Y Guo, C Huang, M Li; (VI) Manuscript writing: All authors; (VII) Final approval of manuscript: All authors.

\#These authors contributed equally to this work.

Correspondence to: Xiaoshun He; Weiqiang Ju; Maogen Chen. Division of Organ Transplant Center, First Affiliated Hospital of Sun Yat-sen University, Guangzhou 510080, China. Email: gdtrc@163.com; weiqiangju@163.com; chenmg3@mail.sysu.edu.cn.

Backgroundk Programmed death protein-1 (PD-1) inhibitors and liver transplantation (LT) are alternative treatments for hepatocellular carcinoma (HCC) patients. The application of PD-1 inhibitors for HCC therapy increases $\mathrm{T}$ cell immune activity, while immunosuppression is required for patients receiving transplantation. More clinical investigation is required to determine methods to balance these treatment effects. In this article, we are the first to describe the clinical characteristics, imaging findings, and outcomes of 5 LT patients who had a history of HCC and received anti-PD-1 therapy.

Methods: Data from 5 patients who were diagnosed with HCC and received LT after PD-1 inhibition were analyzed. The doses and courses of PD-1 and preoperative and postoperative characteristics were compared and analyzed.

Results: The mean interval between PD-1 inhibition and LT was $63.80 \pm 18.26$ days. One patient experienced recurrence in the liver, vertebrae and lungs after 7 months, and 1 patient experienced recurrence in the lungs after 3 months. All patients displayed normal liver function at the latest follow-up visit. No acute allograft rejections occurred in any patient.

Conclusions: PD-1 inhibitors may be safe for the treatment of HCC before LT when the interval between the two treatments is sufficient. Further investigations are needed for to validate these findings.

Keywords: Programmed death protein-1 inhibitor; liver transplantation (LT); acute allograft rejection; hepatocellular carcinoma and case report

Submitted Apr 23, 2021. Accepted for publication Jul 15, 2021.

doi: 10.21037/apm-21-999

View this article at: https://dx.doi.org/10.21037/apm-21-999 


\section{Introduction}

Hepatocellular carcinoma (HCC) is a malignant disease with high morbidity and mortality rates. HCC is the fifth most common cancer worldwide and third most deadly cancer in China (1). There have been tremendous efforts in the development of therapeutic strategies in recent decades. Hepatectomy was once the standard treatment for HCC worldwide. Surgical removal of the tumor is associated with better patient prognosis, but only a small number of tumors are suitable for surgical resection at the patient's first clinical review (2). For more advanced tumors, the rate of recurrence is high after surgery. In addition, patients may not be candidates for surgery when the tumor is large or when distant metastases are present. Molecular targeted drugs and immunotherapy might be an effective option to decrease symptoms of the disease and maximize the duration of survival. The successful application of programmed death protein-1 (PD-1) inhibitors such as nivolumab and pembrolizumab for cancer therapy have led to the expansion of the use of immunotherapy in oncology (3). The efficacy of immunotherapy differs among individuals, and it may not be effective for many patients (4).

Liver transplantation (LT) is considered the most effective treatment for end-stage liver diseases (ESLD), including HCC (5). However, the application of PD-1 inhibitors and LT for cancer patients is controversial. PD-1 and its ligand programmed death ligand-1 (PD-L1) were proven to be essential components of both graft induction and maintenance of immune tolerance $(6,7)$. It has been widely reported that patients with metastatic cancer with a history of liver transplantation, the application of PD-1 inhibition leads to acute allograft rejection (8). For this reason, all clinical trials evaluating PD-1 inhibitors have excluded transplanted patients (9). In addition, prognosis after LT in patients with a history of HCC treated with PD-1 inhibition has not yet been reported. Here, we are the first to describe the clinical characteristics, imaging findings, and outcomes of 5 LT patients who had a history of HCC treated with PD-1 inhibitors.

We present the following article in accordance with the AME Case Series reporting checklist (available at https:// dx.doi.org/10.21037/apm-21-999).

\section{Methods}

\section{Ethical statement}

The study was conducted in accordance with the tenets of the Declaration of Helsinki (as revised in 2013) and approved by the Institutional Ethics Committee for Clinical Research and Animal Trials of the First Affiliated Hospital of Sun Yat-sen University \{Approved ID: 2021[433]\}. All patients gave consent for their details and accompanying images to be published.

\section{General information of participants}

From November 2018 to July 2020, 5 patients who were diagnosed with HCC and received LT after having previously received PD-1 inhibition therapy were enrolled in this study. The PD-1 inhibitor used in this study was nivolumab. Pretransplant data, including sex, age at pretransplant treatment, course and dose of PD-1 treatment, the interval between the last PD-1 treatment and the transplant date, the alpha fetoprotein level, cirrhosis status, Child-Pugh score and model for end-stage liver disease (MELD) score, were recorded and analyzed. In addition, posttransplant conditions, including liver function recovery, acute allograft rejection, recurrence, progressionfree survival (PFS), and overall survival (OS), were also recorded.

\section{Follow-up}

Postoperative visits occurred on postoperative days (PODs) $1-7$, POD 14 and each postoperative month (POM). Routine Doppler ultrasound of the liver graft blood flow and biliary tract was performed once every 2 days for 7 days. The immunosuppressive regimen was tacrolimus (Tac)+mycophenolate mofetil (MMF). Corticosteroids were not included in routine regimens due to their side effects. For deceased patients and patients who experienced disease recurrence, the date of death or recurrence was used as the date of the last follow-up. Biomedical values, tacrolimus concentration, imaging examinations and tumor markers (AFPs) were documented. Afterward, imaging studies were performed based on patients' clinical status or laboratory findings.

\section{Results}

Pretransplant imaging descriptions of 5 recipients are presented in Figure 1, and the clinical characteristics and outcomes of these patients are shown in Table 1. The mean age of the patients was $53.20 \pm 5.42$ years. All patients received pretransplant transcatheter 


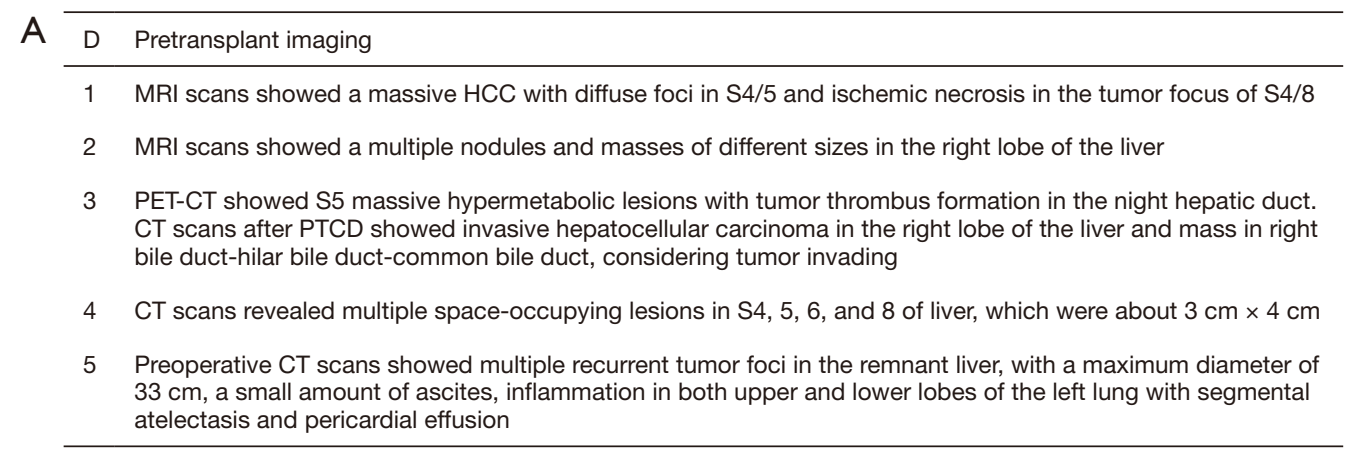

B
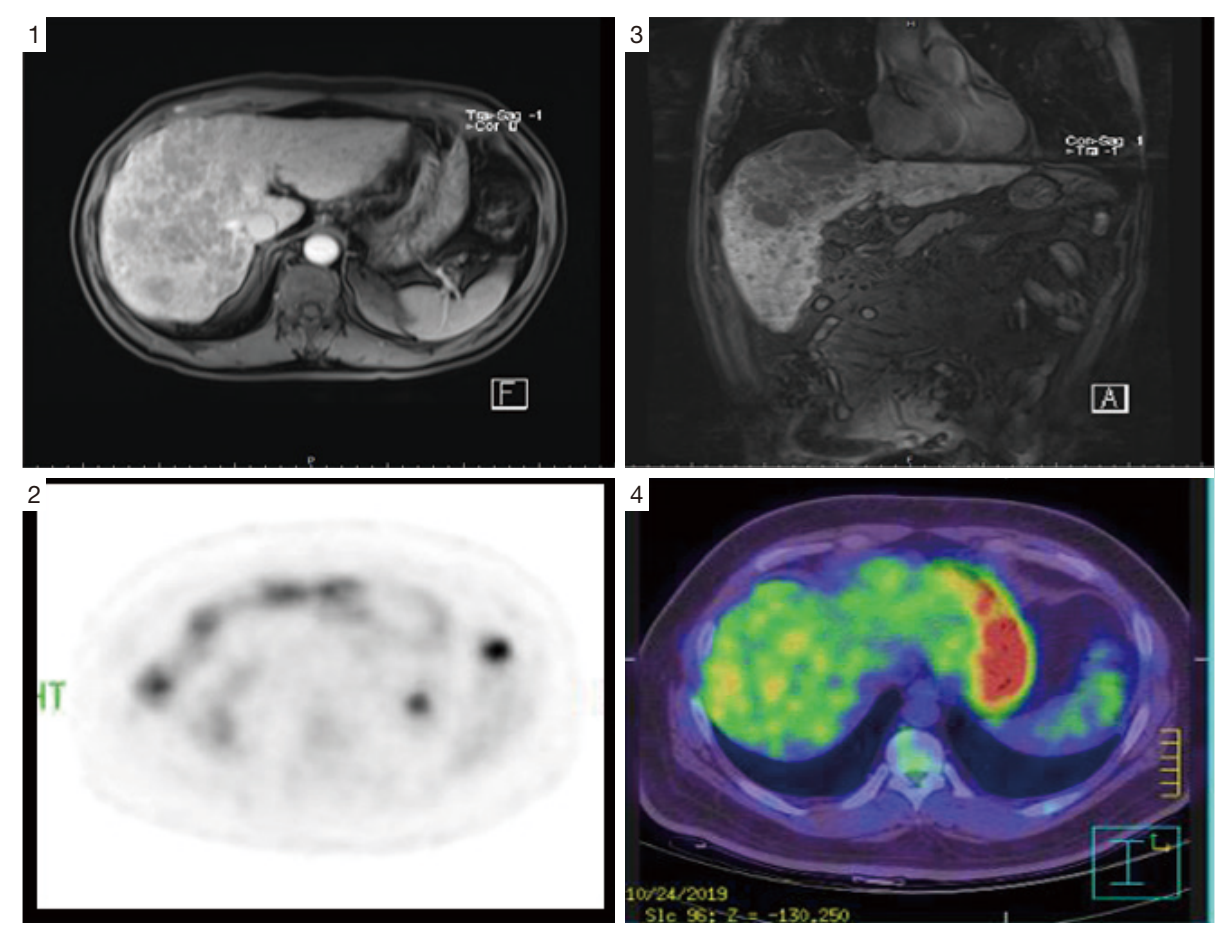

Figure 1 Pretransplant imaging descriptions of 5 patients. (A) The descriptions of all 5 patients' images; (B) images of patient 1 and patient 3.

arterial chemoembolization (TACE), patient 3 received radiofrequency ablation (RFA), and patient 5 received resection. The mean Child-Pugh score and MELD score were $8.60 \pm 1.08$ and $14.00 \pm 3.07$, respectively. The average interval was $63.80 \pm 18.26$ days. The peak alanine aminotransferase (ALT), aspartate aminotransferase (AST), international normalized ratio (INR) and TBil within PODs $1-7$ were $426.00 \pm 183.92,1018.2 \pm 534.64 \mathrm{U} / \mathrm{L}$, $1.73 \pm 0.17$ and $111.06 \pm 39.51 \mathrm{mmol} / \mathrm{L}$, respectively. The postoperative pathological diagnoses of 5 patients are presented in Figure 2, and in patient 3, no clear surviving cancer cells were found. None of the patients experienced acute allograft rejection. Posttransplant AFP monitoring showed that patient 1 and patient 2 presented with elevated
AFP at POM 13 and POM 7, respectively (Figure 3). FK level plots showed that concentrations fluctuated considerably in the early postoperative period in all patients but ultimately leveled off by 12 weeks postoperatively (Figure 4). Two patients developed recurrence after transplantation. Patient 1 experienced relapse with metastatic disease of the liver, vertebrae, and lungs on POM 7 , as confirmed by imaging. He received lenvatinib at a dose of $16 \mathrm{mg}$ every day and 3 courses of radiotherapy to the thoracic vertebrae 12 (T12) at a dose of 24 Gy. Patient 2 experienced relapse with metastatic disease of the lungs on POM 3, as confirmed by imaging. She received lenvatinib at a dose of $16 \mathrm{mg}$ every day. No acute allograft rejections occurred in any patient during the follow-up period. In 
Table 1 Clinical characteristics and outcomes of 5 patients involved in this report

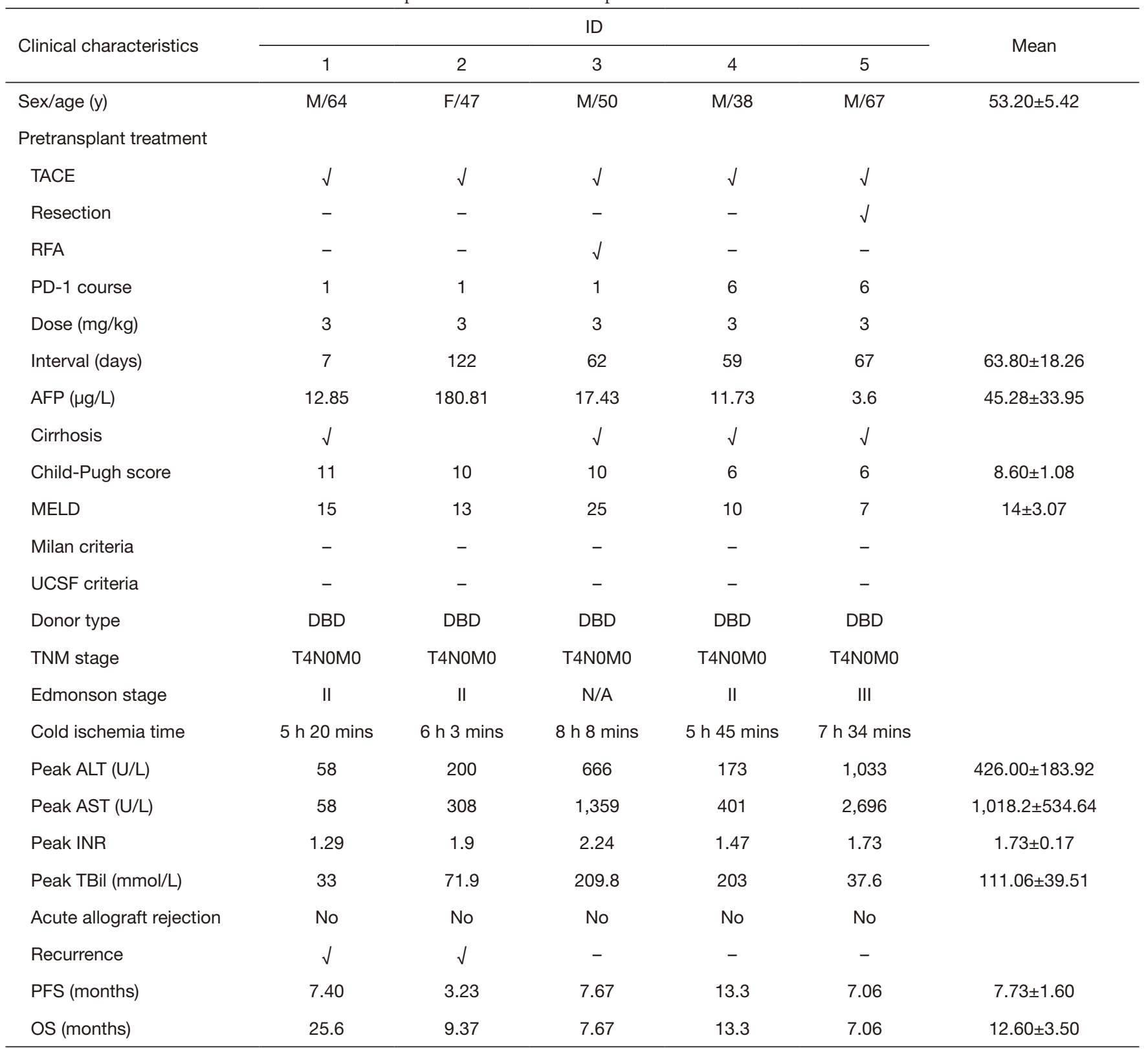

ALT, alanine aminotransferase; AST, aspartate aminotransferase; DBD, donor after brain death; INR, international normalized ratio; MELD, model for end-stage disease; OS, overall survival; PFS, progression-free survival; RFA, radiofrequency ablation; TACE, transcatheter arterial chemoembolization.

addition, all patients displayed normal liver function at the last follow-up date after transplantation.

\section{Discussion}

LT for patients with a history of PD-1 inhibitor therapy needs to be considered with a great deal of caution given the possibility of graft rejection after LT. Moreover, it is unclear how long it is necessary to wait after the application of PD-1 inhibitors to safely perform LT. In this article, we have described the cases of 5 patients to provide preliminary insight into the possible outcomes of LT in patients who have received PD-1 inhibition therapy.

Hepatectomy was once the standard treatment for HCC 


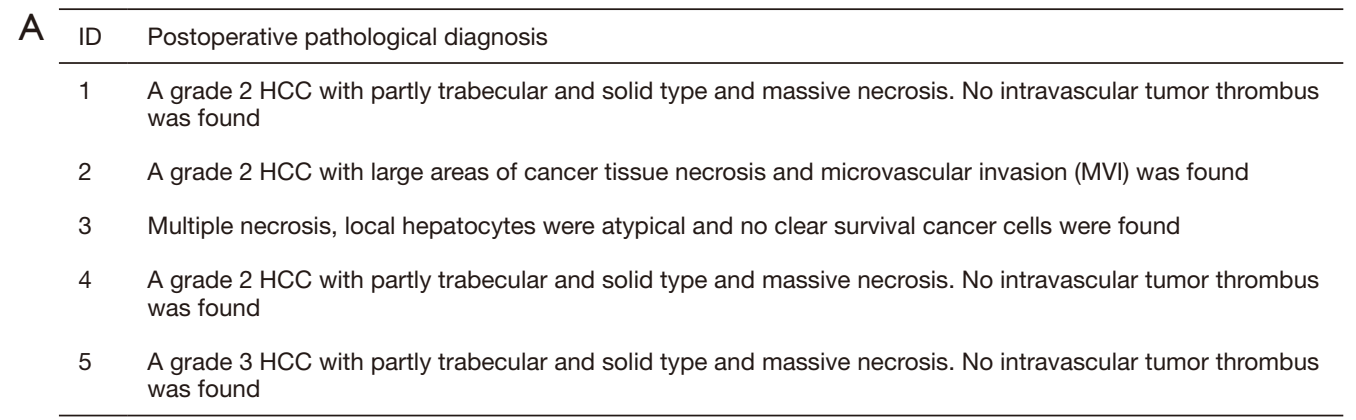

B
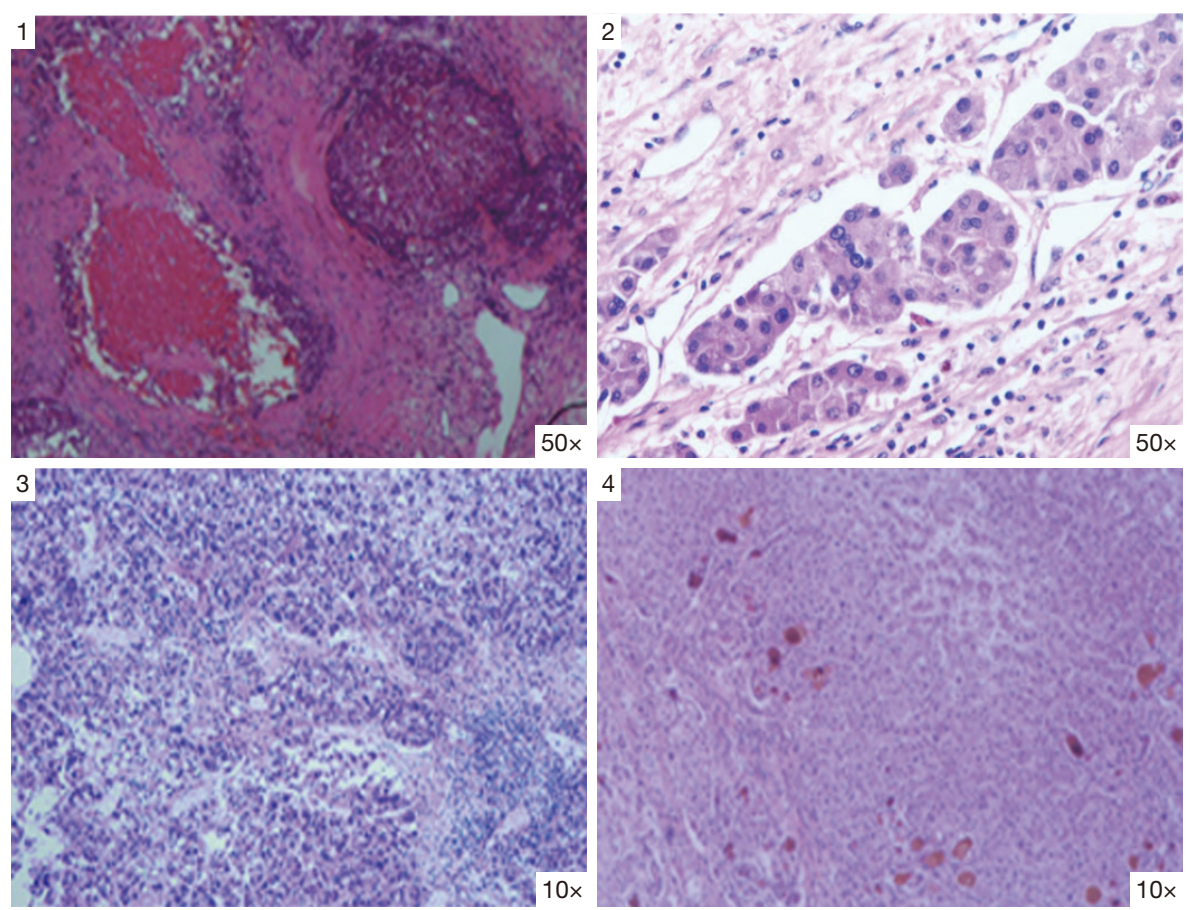

Figure 2 Posttransplant pathological diagnoses of 5 patients. (A) Descriptions of all 5 patients' pathological reports; (B) pathological images with hematoxylin-eosin staining of patients $1,2,3$ and 4 .

worldwide. However, in China, HCC is often diagnosed at the advanced stage of the disease, and there are insufficient highly effective therapies (10). Immunity plays a major role in carcinogenesis, including in HCC. Checkpoint inhibitors, which are novel drugs that enhance the immune system's ability to attack cancer cells, have been successfully introduced as therapies for several malignancies, including HCC (11). Khemlina et al. showed in their review that PDL1 is expressed in $82 \%$ of HCC specimens and that PDL1 expression is higher in hepatitis B-positive patients than in those negative for this virus (12). Several studies have revealed that patients with positive PD-L1 expression have significantly poorer DFS and OS rates than patients who are PD-L1-negative. PD-1/PD-L1 inhibitors are the backbone of HCC treatment. However, in our study, none of the 5 patients obtained a clinical benefit from PD-1 inhibitor therapy. The immunohistochemistry results in patient 1 indicated that the patient was PD- 1 negative. Moreover, there are several reasons why LT could be the most beneficial option for the treatment of some patients. In China, patients tend to undergo surgical resection or conservative treatment due to economic or ideological reasons, even if the tumors are detected early (13). LT is typically considered only when other treatments are ineffective or if the tumor progresses.

The relationship between PD-1 inhibitors and LT is controversial. Biondani et al. reported the first case of an LT patient who had received PD-1 inhibitors (14). In that 

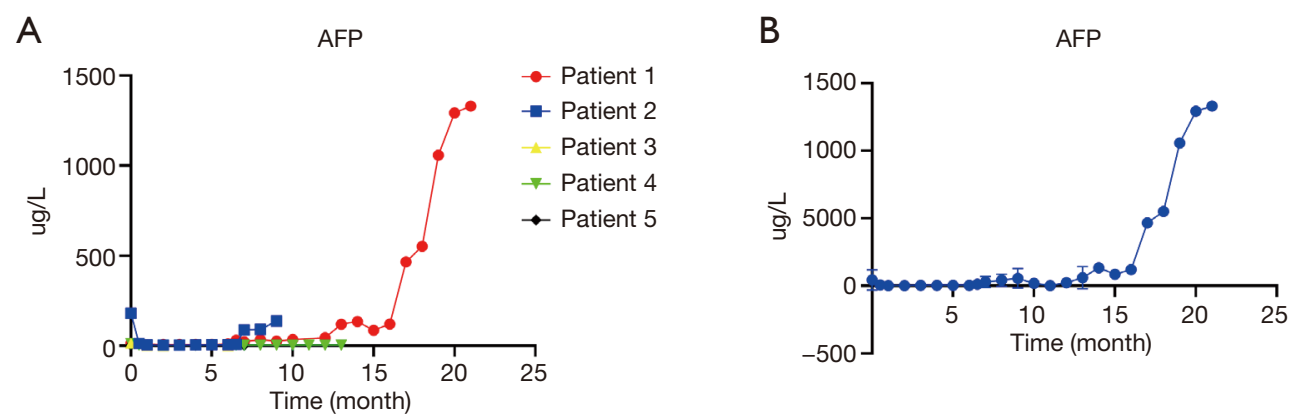

Figure 3 Dynamic changes in AFP levels in 5 patients during the follow-up period. (A) Dynamic changes in each patient; (B) dynamic changes in total. AFP, alpha fetoprotein.
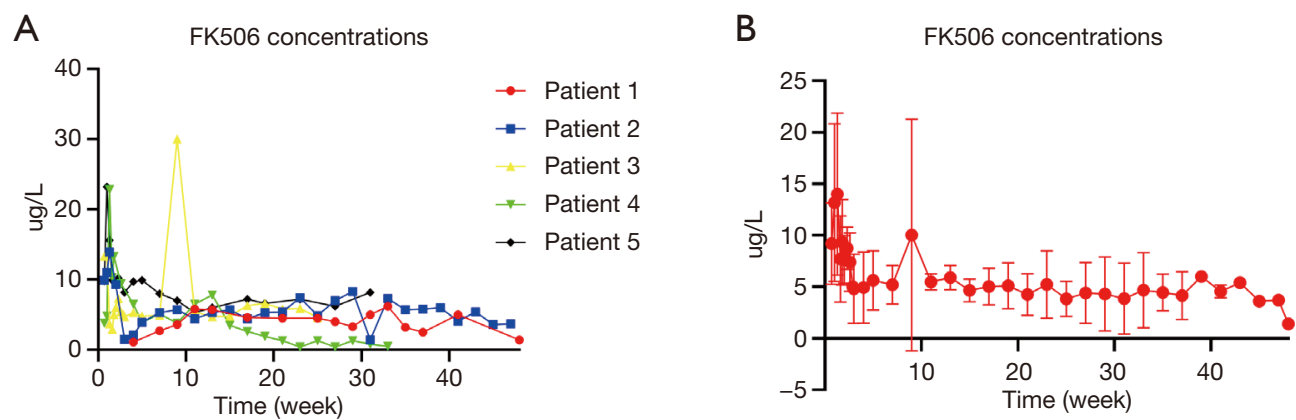

Figure 4 Dynamic changes in FK506 concentrations in 5 patients during the follow-up period. (A) Dynamic changes in each patient; (B) dynamic changes in total.

case, the patient did not respond to nivolumab and did not experience acute allograft rejection. However, in another case report, Friend et al. reported that two LT patients rapidly developed irreversible acute rejection shortly after starting PD-1 therapy and ultimately died (15). DeLeon et al. reported the cases of 7 patients with metastatic cancer with a history of LT who then received PD-1 inhibitor therapy and found that 2 of the 7 patients experienced allograft rejections (16). All these studies indicate that PD-1 should be avoided or used with extreme caution for LT patients. Furthermore, prognosis after LT in patients with a history of HCC treated with PD-1 inhibition has not yet been reported and remains unclear. This is the first report of 5 patients who received LT after immunotherapy with PD-1 inhibitors. No patients had experienced acute allograft rejections in this study. We hypothesize that PD-1 inhibitors may be safe for the treatment of HCC before LT when the interval between the two treatments is sufficient. When the interval is beyond one week, the effect of PD-1 inhibition on the immune status will have dissipated there will be no rejection after LT; thus, we speculate that this approach is safe. However, further investigations are needed to verify this hypothesis.

\section{Conclusions}

In conclusion, PD-1 inhibitors may have the potential to be used in the treatment of HCC before LT when the interval between the two treatments is sufficient. Larger studies are needed to establish the safety of this treatment and provide more convincing results.

\section{Acknowledgments}

Funding: This work was supported by the National Natural Science Foundation of China (No. 81401324, 81770410), the Science and Technology Planning Project of Guangdong Province (No. 2016A020215048), the Guangdong Provincial Key Laboratory of Organ Donation and Transplant Immunology (No. 2020B1212060026), the Guangdong Basic and Applied Basic Research Foundation (No. 2020A1515011557, 2020A1515010903), and the 
Guangdong Provincial International Cooperation Base of Science and Technology (Organ Transplantation), China (No. 2015B050501002).

\section{Footnote}

Reporting Checklist: The authors have completed the AME Case Series reporting checklist. Available at https://dx.doi. org/10.21037/apm-21-999

Data Sharing Statement: Available at https://dx.doi. org/10.21037/apm-21-999

Peer Review File: Available at https://dx.doi.org/10.21037/ apm-21-999

Conflicts of Interest: All authors have completed the ICMJE uniform disclosure form (available at https://dx.doi. org/10.21037/apm-21-999). The authors have no conflicts of interest to declare.

Ethical Statement: The authors are accountable for all aspects of the work in ensuring that questions related to the accuracy or integrity of any part of the work are appropriately investigated and resolved. The study was conducted in accordance with the tenets of the Declaration of Helsinki (as revised in 2013) and approved by the Institutional Ethics Committee for Clinical Research and Animal Trials of the First Affiliated Hospital of Sun Yatsen University (Approved ID: 2021[433]). All patients gave consent for their details and accompanying images to be published. All the organs used in our study were acquired by organ donation, and none were from executed prisoners.

Open Access Statement: This is an Open Access article distributed in accordance with the Creative Commons Attribution-NonCommercial-NoDerivs 4.0 International License (CC BY-NC-ND 4.0), which permits the noncommercial replication and distribution of the article with the strict proviso that no changes or edits are made and the original work is properly cited (including links to both the formal publication through the relevant DOI and the license). See: https://creativecommons.org/licenses/by-nc-nd/4.0/.

\section{References}

1. Chen $\mathrm{W}$, Zheng R, Baade PD, et al. Cancer statistics in China, 2015. CA Cancer J Clin 2016;66:115-32.
2. Cauchy F, Fuks D, Belghiti J. HCC: current surgical treatment concepts. Langenbecks Arch Surg 2012;397:681-95.

3. Yao X, Wang L, Gao J. Overshadowed prospect of programmed cell death protein-1 (PD-1) inhibitor as monotherapy for patients with advanced hepatocellular carcinoma. Biosci Trends 2019;13:282-3.

4. Yang Y. Cancer immunotherapy: harnessing the immune system to battle cancer. J Clin Invest 2015;125:3335-7.

5. Sangiovanni A, Colombo M. Treatment of hepatocellular carcinoma: beyond international guidelines. Liver Int 2016;36 Suppl 1:124-9.

6. Barclay J, Creswell J, León J. Cancer immunotherapy and the PD-1/PD-L1 checkpoint pathway. Arch Esp Urol 2018;71:393-9.

7. Riella LV, Watanabe T, Sage PT, et al. Essential role of PDL1 expression on nonhematopoietic donor cells in acquired tolerance to vascularized cardiac allografts. Am J Transplant 2011;11:832-40.

8. Maggiore U, Pascual J. The Bad and the Good News on Cancer Immunotherapy: Implications for Organ Transplant Recipients. Adv Chronic Kidney Dis 2016;23:312-6.

9. Kittai AS, Oldham H, Cetnar J, et al. Immune Checkpoint Inhibitors in Organ Transplant Patients. J Immunother 2017;40:277-81.

10. El-Khoueiry AB, Sangro B, Yau T, et al. Nivolumab in patients with advanced hepatocellular carcinoma (CheckMate 040): an open-label, non-comparative, phase $1 / 2$ dose escalation and expansion trial. Lancet 2017;389:2492-502.

11. Voutsadakis IA. PD-1 inhibitors monotherapy in hepatocellular carcinoma: Meta-analysis and systematic review. Hepatobiliary Pancreat Dis Int 2019;18:505-10.

12. Khemlina G, Ikeda S, Kurzrock R. The biology of Hepatocellular carcinoma: implications for genomic and immune therapies. Mol Cancer 2017;16:149.

13. Chen Z, Lin X, Chen C, et al. Analysis of preoperative circulating tumor cells for recurrence in patients with hepatocellular carcinoma after liver transplantation. Ann Transl Med 2020;8:1067.

14. Biondani P, De Martin E, Samuel D. Safety of an antiPD-1 immune checkpoint inhibitor in a liver transplant recipient. Ann Oncol 2018;29:286-7.

15. Friend BD, Venick RS, McDiarmid SV, et al. Fatal orthotopic liver transplant organ rejection induced by a checkpoint inhibitor in two patients with refractory, metastatic hepatocellular carcinoma. Pediatr Blood Cancer 
2017. doi: 10.1002/pbc.26682.

16. DeLeon TT, Salomao MA, Aqel BA, et al. Pilot evaluation of PD-1 inhibition in metastatic cancer patients with

Cite this article as: Chen Z, Hong X, Wang T, Guo Y, Huang C, Li M, He X, Ju W, Chen M. Prognosis after liver transplantation in patients treated with anti-PD-1 immunotherapy for advanced hepatocellular carcinoma: case series. Ann Palliat Med 2021;10(9):9354-9361. doi: 10.21037/apm21-999 a history of liver transplantation: the Mayo Clinic experience. J Gastrointest Oncol 2018;9:1054-62. 